\title{
The Causes of Distress in Paediatric Outpatients Receiving Dilating Drops*
}

\author{
Nashila Hirjï, Sophie Jones, Graham Thompson
}

St. George’s Hospital, London, England.

Email: \#nashila@doctors.org.uk

Received January $1^{\text {st }}, 2012$; revised February $19^{\text {th }}$, 2012; accepted March $4^{\text {th }}, 2012$

\begin{abstract}
Background/Aims: Paediatric outpatients are often dilated with cyclopentolate drops. These cause discomfort and distress, which may impede subsequent examination. We aimed to determine the distress caused by cyclopentolate drops, and other factors in the clinic environment. Methods: Over an 8 week period, questionnaires were issued to guardians of all paediatric outpatients aged under 10 years receiving cyclopentolate. The childrens'distress was graded on a scale of 1 - $10(1=$ no distress, $10=$ severe distress $)$. Waiting time and ease of examination were recorded. Data was analysed using Stata statistics, and significant differences were reported at the $\mathrm{P}<0.05$ level. Results: The 72 children were grouped as under 4 years $(n=43$, Group A), aged $4-7$ years $(n=19$, Group B), and aged 7 - 10 years $(n=10$, Group C). Median distress levels at home (baseline), on arrival, on dilation, and on examination were as follows: Group A; 1, 2, 7, 6 respectively; Group B; 1, 1, 6, 2 and Group C; 1, 1, 4.5, 1. All age groups were significantly more distressed on examination compared to baseline. Distress scores on examination were significantly greater for Group A, in keeping with the greatest number of suboptimal examinations. Guardians reported that a prolonged waiting time and bright examination lights also contributed to distress. Conclusions: This study confirms that cyclopentolate causes significant distress in young children, and in $45 \%$ of very young children, the examination is difficult. Proxymetacaine prior to cyclopentolate is a possible solution, but other distressing factors should also be addressed for optimal outcomes.
\end{abstract}

Keywords: Paediatric; Cyclopentolate; Proxymetacaine Hydrochloride; Dilating Drops

\section{Introduction}

Cycloplegic examination is a key element in the assessment of children presenting to ophthalmology outpatients. It is most often achieved using cyclopentolate $0.5 \%$ or $1 \%$ depending on age. However, this drug is disliked by children due to the stinging it produces on instillation. Children receiving the drop commonly become distressed, and their resultant tearful and uncooperative behaviour can lead to significant difficulty in subsequent examination. Additionally, paediatric distress can contribute to a frightening environment in the clinical setting, and may have long-term harmful effects on the doctor-patient relationship.

A previous study demonstrated that instillation of proxymetacaine hydrochloride $0.5 \%$ prior to cyclopentolate resulted in less traumatic cycloplegia in children [1]. This was further supported by a second study, which showed a significant reduction in the total discomfort

\footnotetext{
${ }^{*}$ This study received no specific grant from any funding agency in the public, commercial or not-for-profit sectors.

The authors declare no competing interests.

${ }^{\#}$ Corresponding author.
}

with cyclopentolate administered after premedication with proxymetacaine, compared with the use of cyclopentolate instilled after placebo [2]. However, the authors of this study suggested that other factors may also contribute to the distress experienced by children attending ophthalmology outpatients, and until now, this aspect has remained unexplored.

Since the use of proxymetacaine prior to cyclopentolate is often not standard practice, our study aimed to determine the degree of distress caused by cyclopentolate drops. In addition, we investigated other factors that may lead to distress in the paediatric outpatient setting.

\section{Patients and Methods}

Over an 8 week period, all children under the age of 10 years requiring cycloplegic examination in a paediatric ophthalmology outpatient clinic were assessed. Each received our standard dilating regime of cyclopentolate drops $0.5 \%$ or $1 \%$ (Minims, Smith and Nephew Pharmaceuticals, UK), and phenylephrine 2.5\% drops (Minims, Smith and Nephew Pharmaceuticals, UK) in addition for those children with very dark eyes. Questionnaires were 
issued to the guardians of all children on arrival in clinic. The questionnaires required the guardians to grade the child's distress on a scale of 1 - 10 ( 1 = no distress, $10=$ severe distress), at 4 points during the day: at home (prior to coming to clinic); on arrival in the department; on receiving the cyclopentolate drops; and on examination. The time of arrival and time at which the patient eventually saw the doctor were documented, enabling the waiting time to be calculated. Guardians were also requested to document other factors they felt may have contributed to their child's distress during their visit. In addition, each doctor in the clinic recorded whether the child was easy to examine (adequate examination performed), difficult to examine (suboptimal examination performed), or unexaminable.

Statistical analysis of the data was performed using Stata statistics. All medians with ranges are reported, and significant differences are reported at the $\mathrm{P}<0.05$ level. Signed rank tests were used to compare distress scores at different time points for each age group. A Kruskal Wallis test was performed to compare distress scores between age groups at different time points. A two-sample Wilcoxon rank-sum test was performed to compare the distress score on examination between the different age groups.

\section{Results}

A total of 72 consecutive patients were included in the study (40 male, 32 female). 43 children were aged under 4 years (Group A), 19 children were aged between 4 and 7 years (Group B), and 10 children were aged between 7 and 10 years (Group C). The mean age was 4.4 years (range 2 months - 10 years). Figure 1 illustrates the variation in the level of distress displayed by children at various points during their visit, with the distress level at home taken as the baseline. The median distress levels at home, on arrival, on dilation, and on examination were as follows, with the range for the distress level at each time point shown in brackets: Group A; 1 (1 [1 to 2]), 2 (3 [1 to 4]), 7 (4 [5 to 9]), 6 (5 [3 to 8]) respectively; Group B; 1 (0 [1 to 1$]), 1$ (3 [1 to 4]), 6 (4 [4 to 8]), 2 (3 [1 to 4]) and Group C; 1 (0 [1 to 1$]), 1$ (1 [1 to 2]), 4.5 (3 [3 to 6]), 1 (2 [1 to 3]). In all age groups, distress levels peaked on receiving cyclopentolate, and decreased to a degree by the time they saw the doctor. However, distress scores were significantly greater on examination in comparison to baseline for all age groups $(\mathrm{P}<0.000$ Groups A \& B, $\mathrm{P}<0.047$ Group C). Distress scores were significantly greater in the youngest age group on examination when compared to both older age groups $(\mathrm{P}<0.001)$. Figure 2 summarises the distress levels displayed by the children in the 3 age groups, and illustrates the variance in the distress observed at various points during the visit, and at home prior to arrival. Figure 3 shows the variation in distress level with waiting time. In all age groups, mean distress levels rose with increased waiting time, with this trend being most marked in the youngest age group. Figure 4 demonstrates the proportion of children in each age group deemed by the examining doctors to be "difficult to examine" or "unexaminable". Factors which guardians felt contributed to their child's distress during the visit included discomfort caused by instillation of the dilating drop (83.3\%), a prolonged waiting time (51.4\%), and the use of bright lights during examination (20.8\%).

\section{Discussions}

There is a significant paucity in the literature of studies on paediatric distress with cycloplegic agents. Our prospective clinical observational study demonstrates that the use of cyclopentolate in paediatric patients causes

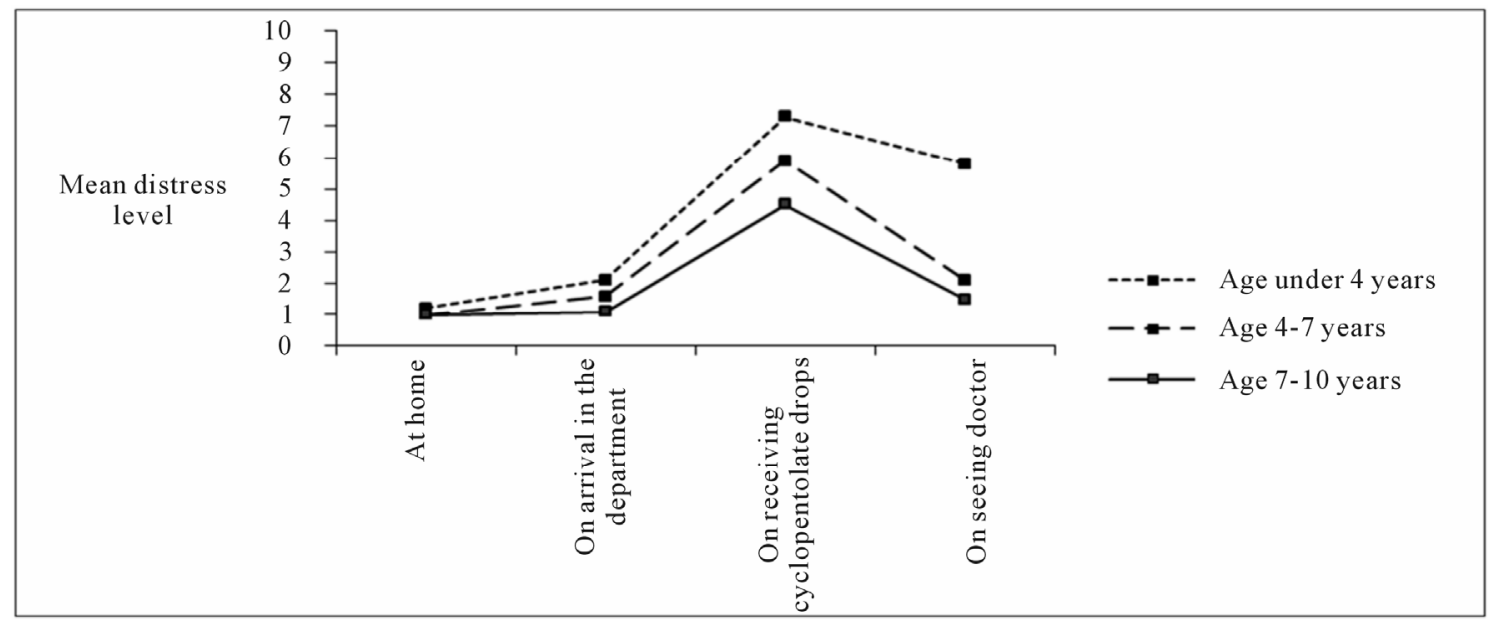

Figure 1. Mean distress levels displayed by the 3 age groups at various points during the visit, and at home prior to arrival. For all age groups, mean distress levels peaked on receiving cyclopentolate drops, and this was most marked in the younger age groups. 


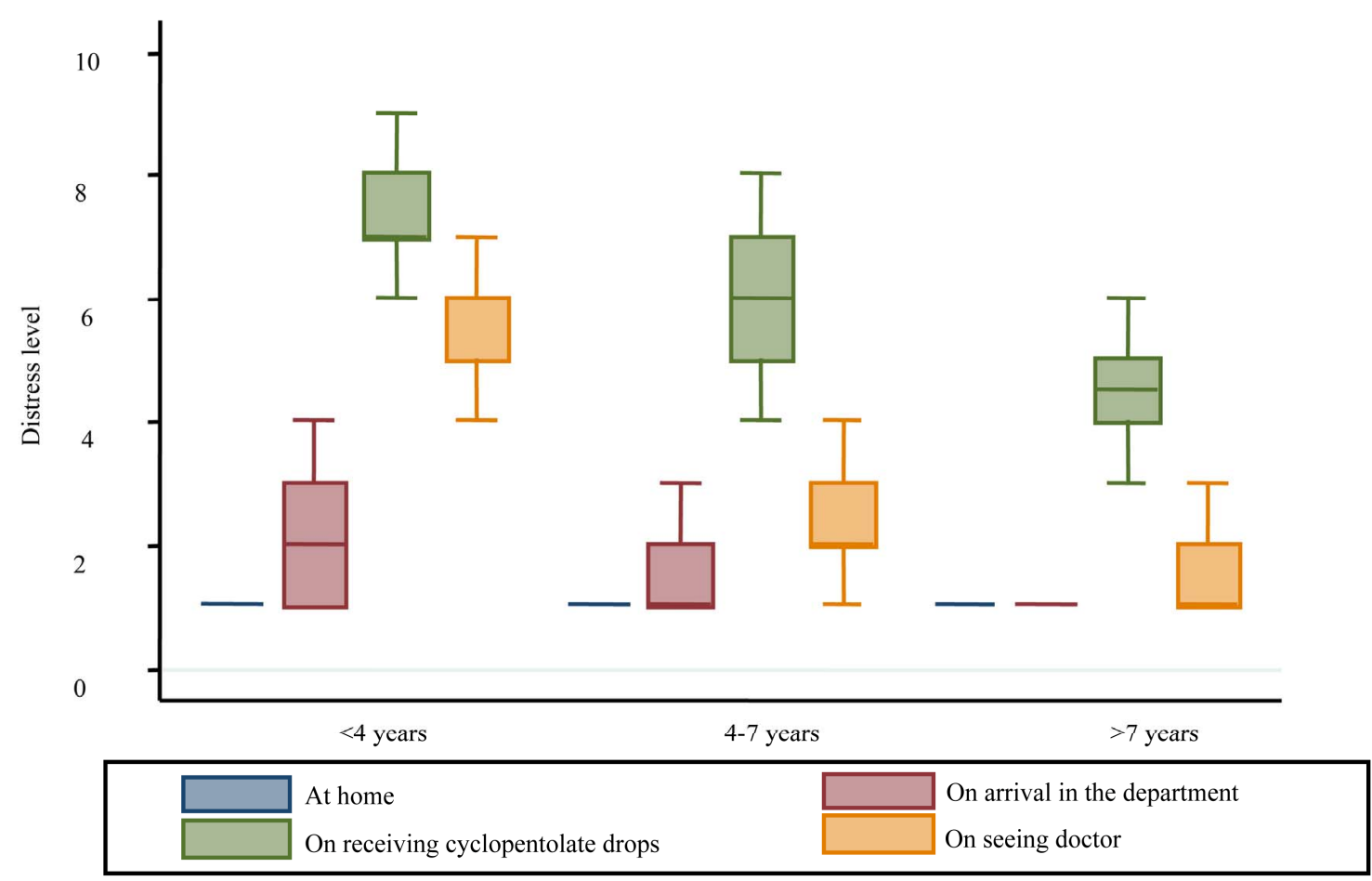

Figure 2. Summary of distress levels displayed by the 3 age groups. The box-and-whisker plot illustrates the variance in the distress levels displayed by the 3 age groups at various points during the visit, and at home prior to arrival. For each set of data, the bottom and top of the box represent the 25 th and 75 th percentile respectively, and the band near the middle of the box represents the median. The ends of the whiskers represent the minimum and maximum values of the data.

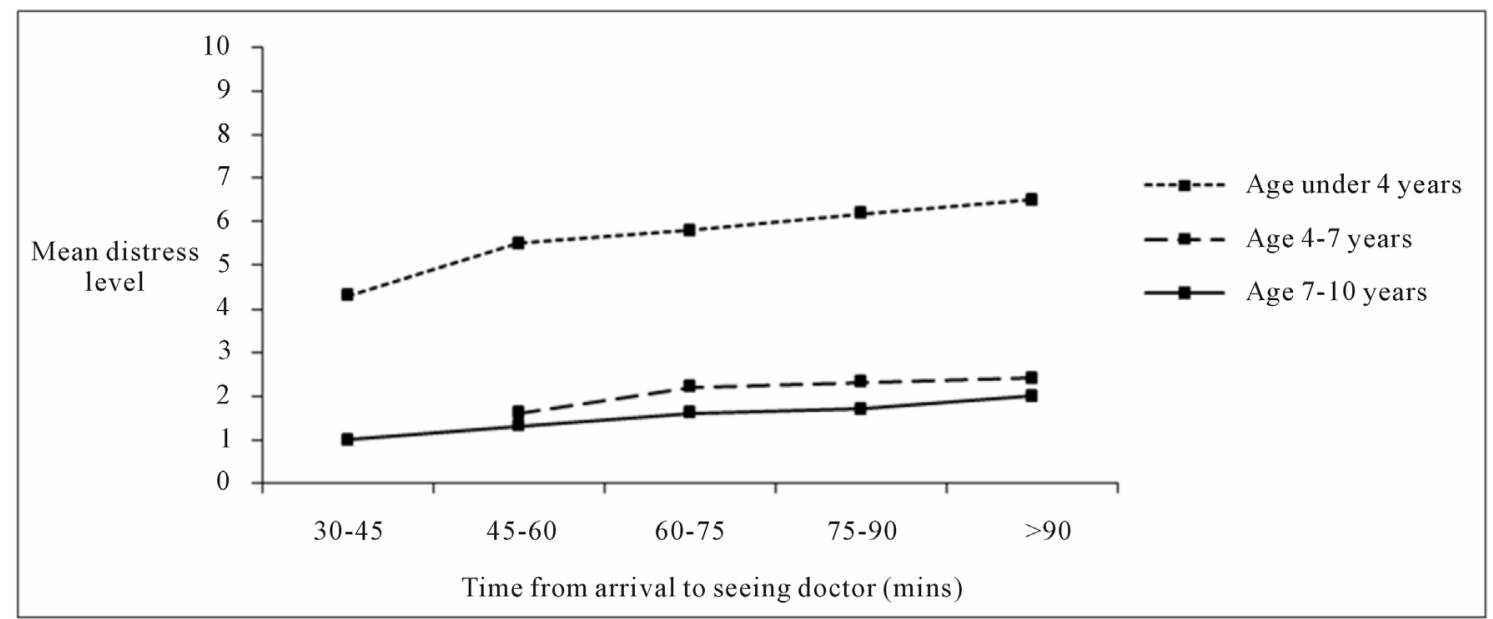

Figure 3. Variation in mean distress levels displayed by the 3 age groups, with increasing waiting time. Across all age groups, mean distress levels rose with increased waiting time. This trend was most marked in children under the age of 4 years.

significant distress. Children in all age groups were significantly more distressed on examination compared to their baseline distress level recorded at home. Furthermore, the distress scores on examination were significantly greater for the youngest age group, in keeping with the greatest number of suboptimal examinations. A suboptimal examination defeats the purpose of the administration of cyclopentolate, and causes unnecessary distress. Of note, the 3 children recorded as "difficult to examine" or "unexaminable" in the 4 - 7 year age group were all under the age of 5 years. To our knowledge, there are no previously established scales for grading paediatric distress on the instillation of eye drops, hence the need for us to create our own in this study. With regard to their reliability, our scales had a subjective element in that guardians may vary in their opinions as to what defined a particular level of distress. However, this variable was controlled to an extent by the fact that each 


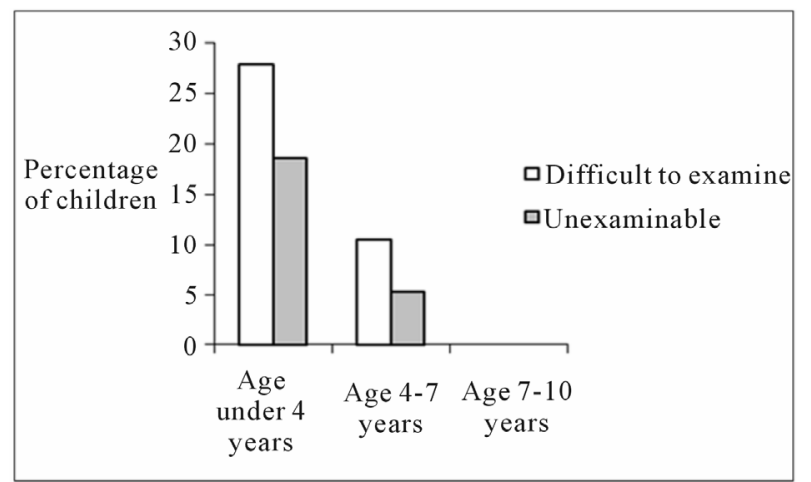

Figure 4. Proportion of children in each age group deemed to be "difficult to examine" or "unexaminable" by the examining doctor. A relatively large proportion of children under the age of 4 years could not be satisfactorily examined. A smaller, though still significant, proportion of children aged 4 - 7 years underwent a suboptimal examination.

guardian rated their child's distress through the course of the visit, therefore changes in distress levels for each particular child could be reported fairly accurately. With this in mind, there appeared to be a definite trend for an increase in distress with instillation of the drops, across age groups. We accept that manifestations of distress may vary amongst children of different ages, making it difficult to make direct comparisons of distress between age groups. However, the practical outcome of difficulty with the examination following the instillation of dilating drops is our main finding in this study.

It is our opinion that the instillation of eye drops in children is a skill, and some individual practitioners are much better than others. Drops should be instilled quickly and with confidence, and not provide a major discussion point in the examination. In our clinics, drops are instilled by orthoptists at the end of their examination, or by a specific paediatric ophthalmic nurse. The same staff was used throughout our study.

Our work might support earlier studies advocating the use of proxymetacaine as a premedication prior to the instillation of cyclopentolate, in an attempt to reduce discomfort $[1,2]$. This would be particularly beneficial in younger children. The discomfort caused on instillation of proxymetacaine is less than that caused by cyclopentolate [2]. It has a rapid action, anaesthetising the eye in less than 30 seconds, with an effect that lasts for 10 - 25 minutes [3]. This would allow for multiple instillations of cyclopentolate within a given time period if necessary. However, there is a potential risk of ocular injury as a result of the child rubbing his or her eyes after the instillation of proxymetacaine, and the anaesthetic is known to have a higher incidence of contact allergy than other topical anaesthetic agents [4]. Furthermore, as a premedication, proxymetacaine would constitute an additional drop administered to the child, and if he or she became distressed on its instillation, the chance to administer the dilating drop may then be lost. Therefore, whilst proxymetacaine prior to cyclopentolate may prove beneficial in reducing paediatric distress in clinic, it is not an ideal solution. Two relatively recent studies have advocated the use of cyclopentolate spray as opposed to drops to achieve mydriasis in children [5,6]. These studies have demonstrated that both cyclopentolate spray and drops have equal cycloplegic efficacy, but that the spray is better tolerated by patients. Although most ophthalmic units still use the traditional cyclopentolate drops in children, a move to the use of the spray formulation may be warranted if this can reduce paediatric distress, without the need for the administration of an additional drug such as proxymetacaine. Our study highlights other factors which may also contribute to paediatric distress in the clinic setting. These include a prolonged waiting time and excessive use of bright lights by examining doctors. Whilst a long wait is often unavoidable for patients attending clinic, the resultant boredom and frustration for paediatric patients may be alleviated by modifying the waiting area to make it better suited to the needs of children, and enlisting the services of multi-disciplinary team members such as paediatric nurses and play specialists during the clinics to help maintain a positive environment. Increasing awareness amongst doctors in paediatric clinics about the distress caused by excessive bright lights may encourage them to use the minimum duration and intensity of light necessary for an adequate examination, leading ultimately to a better assessment of the patient.

In summary, distress amongst paediatric outpatients is multifactorial. Whilst a significant degree of the distress may be attributable to the use of cyclopentolate, the use of proxymetacaine as a premedication has its disadvantages, and is not a perfect or sole solution. Our work demonstrates that there are other factors which may contribute to paediatric distress, and addressing these may create a more positive experience for patients, their guardians, and the healthcare professionals. Where possible, a multifaceted approach should be employed to manage distress amongst paediatric patients, so as to ensure that the chances of performing a meaningful fundal examination and refraction are maximised, and the trust between the child and healthcare professionals is maintained.

\section{REFERENCES}

[1] P. Shah, A. S. Jacks and G. G.Adams, "Paediatric Cycloplegia: A New Approach,” Eye, Vol. 11, No. 6, 1997, pp. 845-846. doi:10.1038/eye.1997.216

[2] M. S. Sutherland and J. D. Young, "Does Instilling Proxymetacaine before Cyclopentolate Significantly Reduce 
Stinging? The Implications of Paediatric Cycloplegia,” British Journal of Ophthalmology, Vol. 85, No. 2, 2001, pp. 244-245. doi:10.1136/bjo.85.2.238g

[3] C. W. Boozan and I. J. Cohen, "Ophthaine, a New Topical Anesthetic for the Eye," American Journal of Ophthalmology, Vol. 36, No. 11, 1953, pp. 1619-1621.

[4] G. O. Rosenwasser, "Complications of Topical Ocular Anesthetics,” International Ophthalmology Clinics, Vol. 29, No. 3, 1989, pp. 153-158.

doi:10.1097/00004397-198902930-00005
[5] C. Y. Wong, D. S. Fan, C. B. Yu and D. S. Lam, "Topical Mydriatic and Cycloplegic Spray for Chinese Children," Journal of Pediatric Ophthalmology and Strabismus, Vol. 40, No. 6, 2003, pp. 349-352.

[6] A. Chafai, S. Ajdnik, L. Lejeune and M. Cordonnier, "Comparative Study of Cyclopentolate Drops versus Spray in Cycloplegia in Children,” Journal Français d'Ophtalmologie, Vol. 29, No. 8, 2006, pp. 896-899. doi:10.1016/S0181-5512(06)70110-3 